\title{
Electric Springs with Coordinated Battery Management for Reducing Voltage and Frequency Fluctuations in Microgrids
}

\author{
T. Yang, Student Member, IEEE, K. T. Mok, Student Member, IEEE, S. C. Tan, Senior Member, IEEE \\ C. K. Lee, Senior Member, IEEE, and S. Y. (Ron) Hui, Fellow, IEEE
}

\begin{abstract}
Electric springs based on power electronics have been proposed as a demand response method for stabilizing power grid fed by substantial intermittent renewable energy sources. Associated with energy storage, they can provide both active and reactive power compensation. Due to the limited storage capacity of the battery, this project explores a new control scheme for the third version of the electric springs (ES3) to operate under the physical constraints of the state-ofcharge of the battery for microgrid stability applications. The ES-3 is based on a bi-directional grid-connected power converter with a battery bank. Unlike the traditional control of grid-connected power inverters for injecting renewable power to the power grid, the proposed control scheme puts the stability of the power grid as a high priority while maintaining its normal bi-directional power flow functions. Such a scheme has been tested in an experimental prototype and a power grid simulator. Results are presented in this paper to illustrate the use of the scheme in battery's monitoring, charging/discharging management, and output power control.
\end{abstract}

Index Terms-Electric spring, battery management system, voltage control, frequency control.

\section{INTRODUCTION}

The increasing penetration of renewable energy of intermittent nature has triggered new concerns about power system stability. The traditional control paradigm of having power generation to follow power demand has to be reversed. Future power grids must have the loads adaptive to the availability of power generation, particularly when renewable power such as wind and solar power becomes substantial. Researchers in the power systems community have responded to this new challenge with various demand-side management methods such as scheduling of delay-tolerant power demand tasks [1], use of energy storage to alleviate peak demands [2], real-time pricing [3] and direct load control or on-off control of electric loads [4].

Energy storage has been suggested as a solution to tackle the power imbalance issue of power generation and load demand in view of the emergence of microgrids with intermittent renewable energy sources. In [5], a microgrid formed of parallel line-interactive uninterruptible power supplies (UPS) has been investigated. Battery-based UPS have been incorporated to provide the voltage source when the microgrid enters the islanding mode. The state-of-charge (SOC) range from $30 \%$ to $100 \%$ of the battery has been considered in the operation of the UPS inverters which also provide virtual inertia through the droop method. This SOC model is also used in [6] in which a decentralized control of a two-area storage-based power system (with diesel generators and batteries to provide long-term and short-term power demand) is studied. The SOC model considered in [5] and [6] is a linear one. In a study of using a hybrid storage (with supercapacitors in parallel with batteries) [7], a simple and linear battery model is used to evaluate the effects of smoothing out the transients of pulsed loads. Battery-based photovoltaic (PV) system [8] and wind power system [9] have also been considered. In [8], the battery is regarded as an ideal voltage source, while a simple equivalent circuit model is adopted in [9].

In order to tackle the possible voltage and stability issues arising from the increasing use of intermittent renewable energy in the distribution networks, researchers in the power electronics community have responded with an alternative approach known as electric springs (ES) [10]. ES can be considered as active suspension systems in the electrical regime. Unlike traditional FACTS devices that primarily deal with reactive power compensation, some versions of ES are associated with non-critical loads, forming smart loads that use active power adaptively according to the availability of power generation. Such a feature automatically plays a constructive role in achieving the new control paradigm of having the power demand following power generation for smart grids. Three versions of ES have been reported so far. The simplified control schematics of the first and third versions of ES are shown in Fig. 1 [10]. The first generation of ES (ES-1) does not involve battery storage. It uses reactive power to stabilize the local mains voltage and adjusts the noncritical load power to balance power supply and demand. The version-two (ES-2) utilizes batteries as energy storage, which allows the ES to output/absorb both active/reactive power [11]. In other words, the ES-2 is able to not only regulate both voltage and frequency of the grid, but also participate in power factor correction and load compensation [12]. ES-3 applies to bidirectional AC/DC converter with battery storage and without association with non-critical load [13], [14].

In this paper, a new control scheme for the active power control of ES-3 with energy storage capacity constraints is described and practically evaluated. In Section II of this paper, a practical battery model used for the SOC estimation is presented. Section III describes the design of the coordinated battery management control for ES-3. Section IV provides the experiment and simulation results. Finally, the conclusion is included in Section V.

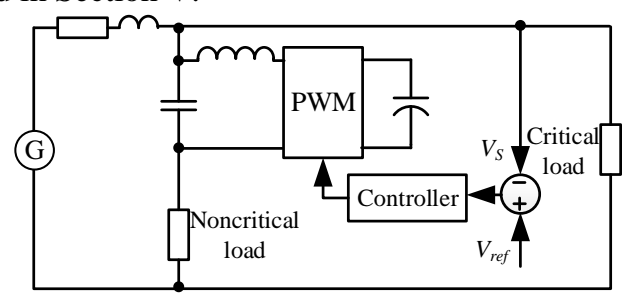

(a)ES-1 


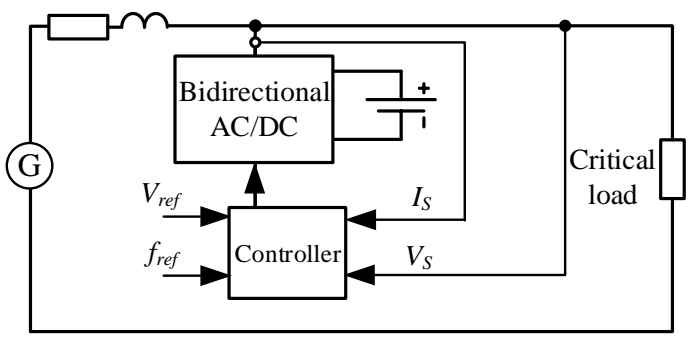

(b)ES-3

Fig. 1. Simplified control schematics of (a) ES-1 and (b) ES-3.

\section{BATTERY MODEL}

Sophisticated battery models can generally be classified into two types, namely the electrochemical models [15]-[17] and equivalent circuit models (electrical models) [18]-[20]. While the electrochemical models are based on the physical and chemical processes of the batteries, the equivalent circuit models have an advantage of being easy to be understood by electrical engineers and easy to be incorporated into circuit simulation. The equivalent circuit models for dynamic battery response prediction are mostly Thevenin-based, using SOCdependent voltage source and RC circuits. However, many of such equivalent circuit models focus too much on dynamic voltage-current performance but ignore the nonlinearity of the batteries' capacity, such as the charge recovery effect [18], [19].

Among various equivalent circuit battery models, the hybrid model [20] shown in Fig. 2 has been chosen for this work. The hybrid model combines the equivalent circuit model and a kinetic battery model. It tracks the nonlinear charge recovery behavior while retaining the dynamic voltage response of the battery. In summary, as the SOC is an essential feedback signal for both the battery model and the coordinated control of ES, such hybrid model could achieve relatively better accuracy than common equivalent circuit models, and then improve the overall performance of the ES.

In this work, the battery parameters extracted from priori experimental data have been incorporated into the DSP controller and Simulink model for experiment and simulation. With the battery model developed, the SOC will be used as a feedback signal for the coordinated operation with the ES. From [20], the key equations of this model are shown as (1) and (2),

$$
\operatorname{SOC}(t)=S O C_{0}-\frac{\left[\int I(t) d t+C_{u n}(t)\right]}{C_{\text {max }}}
$$

where $I, S O C_{0}$ and $C_{\text {max }}$ are the discharging/charging current (positive when discharging), the initial value of SOC and the full capacity of the battery, respectively; $C_{u n}$ is the unavailable capacity of battery, given by,

$$
C_{u n}(t)=\left\{\begin{array}{cc}
C_{u n}\left(t_{0}\right) \mathrm{e}^{-k^{\prime\left(t-t_{0}\right)}}+(1-c) \frac{I}{c} \cdot \frac{1-\mathrm{e}^{-k^{\prime}\left(t-t_{0}\right)}}{k^{\prime}}, t_{0}<t<t_{d} \\
C_{u n}\left(t_{d}\right) \mathrm{e}^{-k^{\left(k t-t_{d}\right)}}, t_{d}<t<t_{r}
\end{array}\right.
$$

where $t_{0}, t_{d}$, and $t_{r}$ are the start time of discharging, the end time of discharging, and the rest time of the battery, respectively; $c$ is the ratio of the available capacity to the full capacity; $k$ ' is a constant to be extracted by experiment.
Equation (2) could be transformed into an incremental equation, which is more convenient in coding for DSP, as shown as (3),

$$
C_{u n}\left((n+1) T_{s}\right)=C_{u n}\left(n T_{s}\right) \cdot e^{-k^{\prime} T_{s}}+\frac{1-e^{-k^{\prime} T_{s}}}{k^{\prime}} \cdot \frac{1-c}{c} I\left(T_{s}\right), n \geq 0
$$

where $T_{s}$ is the sample time. Hence, the SOC of the battery cell could be tracked by (1),

The electrical circuit parameters are the functions of SOC as shown as (4),

$$
\left\{\begin{array}{l}
V_{O C}(S O C)=a_{0} e^{-a_{1} S O C}+a_{2}+a_{3} S O C-\mathrm{a}_{4} S O C^{2}+\mathrm{a}_{5} S O C^{3} \\
R_{\text {series }}(S O C)=b_{0} e^{-b_{1} S O C}+b_{2}+b_{3} S O C-b_{4} S O C^{2}+b_{5} S O C^{3} \\
R_{\text {trans } \_}(S O C)=c_{0} e^{-c_{1} S O C}+c_{2} \\
C_{\text {trans }}(S O C)=d_{0} e^{-d_{1} S O C}+d_{2} \\
R_{\text {trans }-L}(S O C)=e_{0} e^{-e_{1} S O C}+e_{2} \\
C_{\text {trans } L}(S O C)=f_{0} e^{-f_{1} S O C}+f_{2}
\end{array}\right.
$$

where $a_{n}, b_{n}, c_{n}, d_{n}$ and $e_{n}$ are parameters going to be extracted from experiments.

The state-of-health ( $\mathrm{SOH})$ and aging of a battery are not included in the mentioned equations, since this model is for dynamic rather than a long term performance prediction. Such influence could be treated as constants in the extraction process. Also, the influence of the temperature is ignored in the following experiments because all the experiments are conducted in the condition of $24^{\circ} \mathrm{C}$.

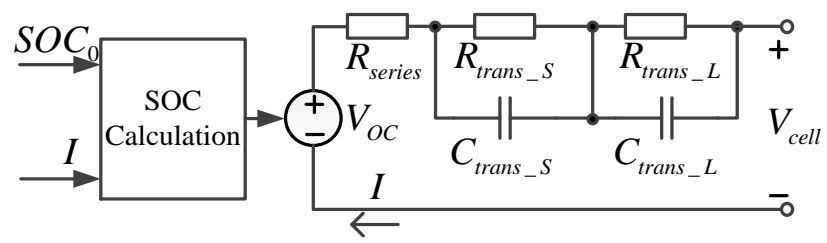

Fig. 2. Hybrid battery model.

\section{COORDINATED BATTERY MANAGEMENT SYSTEM ADPTED IN ELECTRIC SPRINGS}

Unlike many other grid-connected power inverters which inject active power at unity power into the power grid regardless of the voltage and frequency situations, the ES is primarily designed to provide active and reactive compensation for mitigating voltage and frequency fluctuations in power grids in the face of substantial penetration of intermittent renewable energy sources. Due to the limited capacity of batteries, it is necessary to consider the SOC variation so that the battery will not be overcharged or over-discharged. The original control scheme of the ES-3 is shown in Fig. 3. The active and reactive power reference in the control of the ES, namely $P_{r e f}$ and $Q_{r e f}$, are decoupled adopting the direct-quadrature-zero (dpo) transformation, where

$$
\left\{\begin{array}{l}
i_{d_{-} r f}=2 \frac{P_{r e f} v_{d}+Q_{r e f} v_{q}}{v_{d}{ }^{2}+v_{q}{ }^{2}} \\
i_{q_{-} r f}=2 \frac{P_{r f} v_{q}-Q_{r f} v_{d}}{v_{d}{ }^{2}+v_{q}{ }^{2}}
\end{array}\right.
$$


The utility frequency reference $f_{\text {ref }}$ and grid voltage reference $V_{s_{-} r e f}$ are given to the control in such a way that the frequency regulation and voltage stabilization is handled by $P_{r e f}$ and $Q_{r e f}$, respectively.

Fig. 4 shows the simulation results of a microgrid with an ES that uses the original control scheme as shown in Fig. 3. In this simulation, the loading power at the demand side is increased by 0.12 p.u. at $t=4 \mathrm{~s}$. Fig. 4(b) indicates that the ES has to provide active power from $0 \%$ to around $75 \%(0.037$ p.u.) during the transient in order to regulate the frequency at $50 \mathrm{~Hz}$. After the transient, the ES is still continuously delivering an active power of around $60 \%$ (0.03 p.u.) due to the integration function of the PI compensator. If such an approach is adopted, the battery will be fully discharged in a long run if there is no mechanism to manage the active power of the ES. A fully discharged battery cannot provide active power for the power grid. On the other hand, if the battery has to absorb energy, it will be fully charged in the long run. A battery with $100 \%$ SOC cannot absorb more energy. A battery with SOC of $0 \%$ or $100 \%$ cannot be used for discharging and absorbing energy respectively for reducing voltage and frequency fluctuation in a microgrid. Therefore, a modified control scheme for the ES with coordinated BMS is proposed in this work to resolve this issue. The schematic of this modified control is shown in Fig. 5.

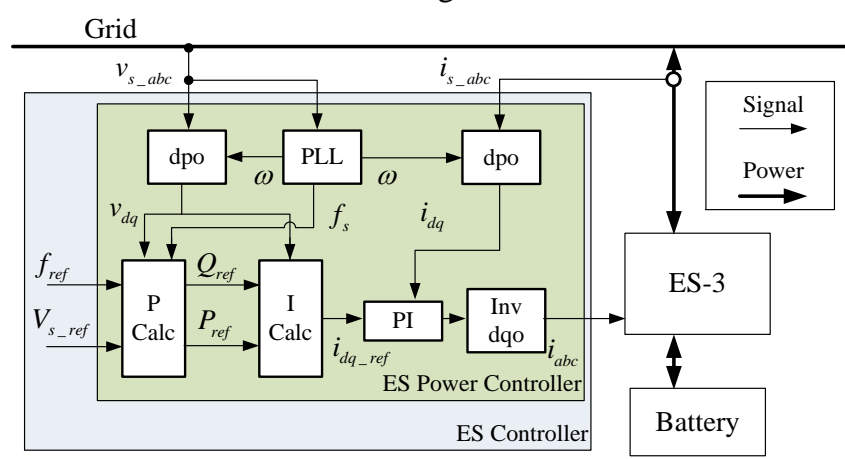

Fig. 3. Original control scheme of ES-3.

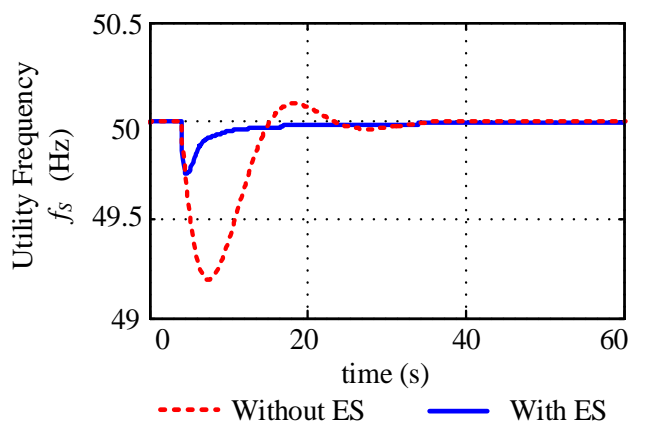

(a) Utility frequency

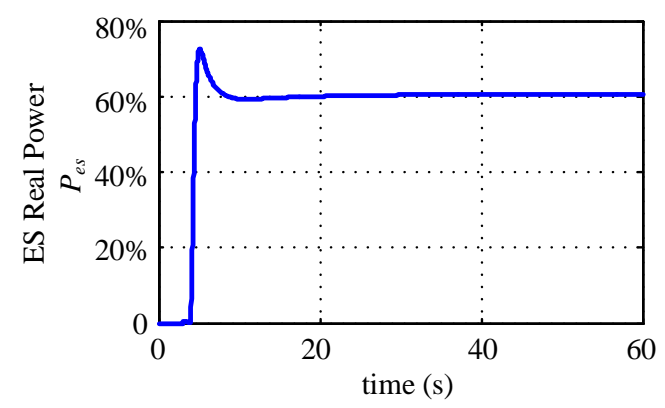

(b) ES active power.

Fig. 4. Simulation results of a microgrid with an ES installed. A step load power increased at $\mathrm{t}=4 \mathrm{~s}$.
In the proposed control scheme, an instant SOC signal $\left(S O C_{f}\right.$, which is normalized from 0 to 1$)$ is introduced. A SOC reference of $S O C_{r e f}=0.5$ is set as a long term target to maintain the battery at $50 \%$ SOC so that it is readily available to either absorb or deliver the active power for the power grid regulation purpose. There are two factors to determine the active power reference of the ES-3, namely $P_{f}$ and $P_{S O C} . P_{f}$ is a signal that controls the amount of active power delivered or adsorbed for the regulation of utility frequency while $P_{S O C}$ is a signal that controls the SOC of the battery storage system. The actual active power reference signal $P_{r e f}$ is the weighted sum of these two signals. Hence,

$$
P_{r f}=a P_{s o c}+(1-a) P_{f}
$$

where $a$ is the weighted factor. It should be noted when $a=0$, the BMS is deactivated, namely $P_{\text {ref }}$ equals to $P_{f}$, and the active power control scheme is the same as the original scheme (as shown in Fig. 3). The adaptive weighted factor $a$ provides a way to coordinate between the battery management and the frequency regulation process, which is defined as a function depending on the absolute SOC error signal $\left(S_{O} O C_{e}=S O C_{r e f}-\right.$ $S O C_{f}$, i.e. the deviation between the SOC reference and the instant SOC status). It can be expressed as

$$
a=f\left(k,\left|S O C_{e}\right|\right)=\frac{e^{2 k|S o c|-k}-e^{-k}}{1-e^{-k}}
$$

where $k$ is a parameter which determines the profile of $a$ as shown in Fig. 6. This function ensures that the charging and discharging of the battery can be performed exponentially in a way that the charging/discharging rate is relatively faster when the absolute SOC error is large while it is slower when the instant status of the SOC closes to the reference value $S O C_{r e f}$. The choice of $k$ depends on the configuration of the system and the design requirements. A higher value of $k$ results in a wider range of the SOC error, as well as a more aggressive charging/discharging manner when the SOC is getting closer to the upper and lower limits of the working region. Compared with some other SOC control schemes, the proposed method not only provides an adaptively weighted priority between the mitigating of grid fluctuation and maintaining the storage system within the healthy SOC range, but also avoids abrupt change of output power for SOC control during transient operations [21].

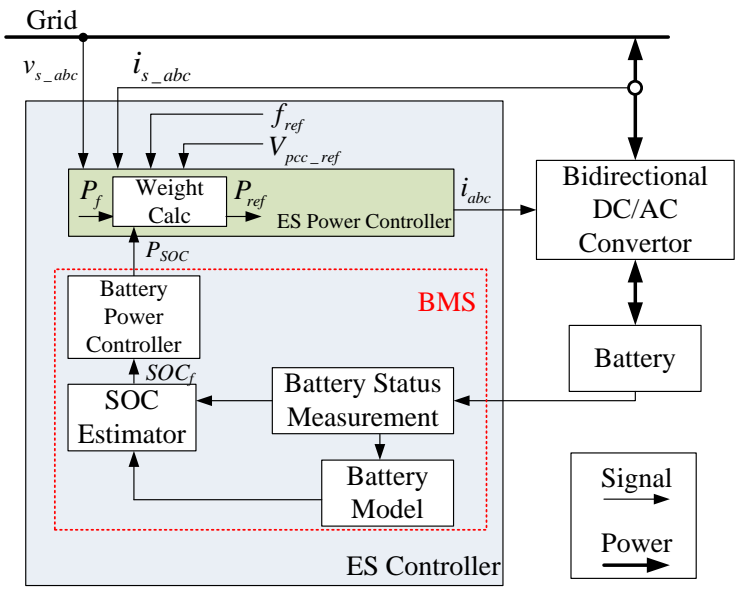

Fig. 5. New control scheme of ES-3 with the coordinated BMS. 
The proposed control scheme has two modes of operation on active power control, namely the active mode and reset mode. During the active mode, active power of the ES is mainly used to regulate the utility frequency to its nominal value within a deviation of $\Delta f_{d z \_ \text {low }}$ (Fig.7). During the reset mode, the control loop gradually reduces the absolute value of $P_{f}$ to 0 , which helps the $P_{S O C}$ to maintain the SOC to 0.5 .

A dead zone control block with a hysteresis band is used to determine the mode of the operation. The dead zone control block defines two frequency deviation ranges, namely $\Delta f_{d z \_l o w}$ and $\Delta f_{d z \_ \text {high }}$ as shown in Fig 7. The control enters the active mode if the frequency error $f_{e}$ exceeds the range of $\Delta f_{d z}$ high . In contrast, the control returns from the active to the reset mode if $f_{e}$ is reduced to a value within the range of $\Delta f_{d z}$ low. The hysteresis band, namely the gap between $\Delta f_{d z \_h i g h}$ and $\Delta f_{d z \_l o w}$, provides a smooth transition of both modes and prevents mode-switching oscillation. In the simulation and experiment, $\Delta f_{d z \_ \text {low }}$ is set to $\pm 20 \mathrm{mHz}$, which is a typical dead-band of the primary frequency control of governors [22]. The modified frequency control scheme is shown in Fig. 8.

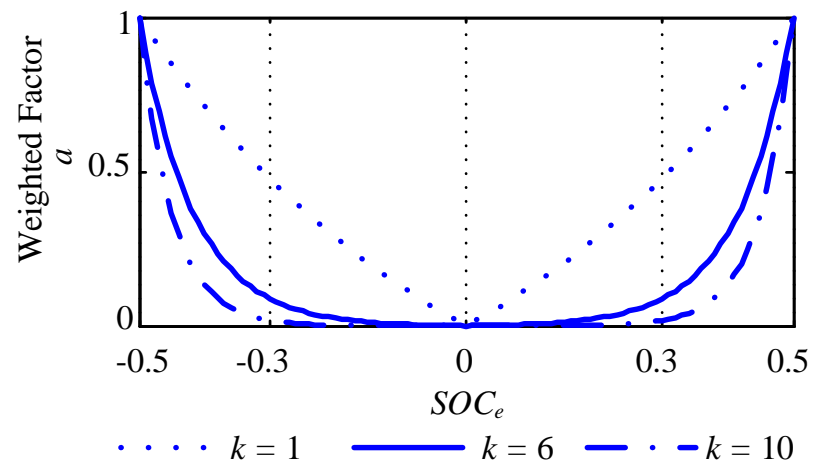

Fig. 6. The weighted factor $a$ versus the $S O C_{e}$ with different values of $k$.

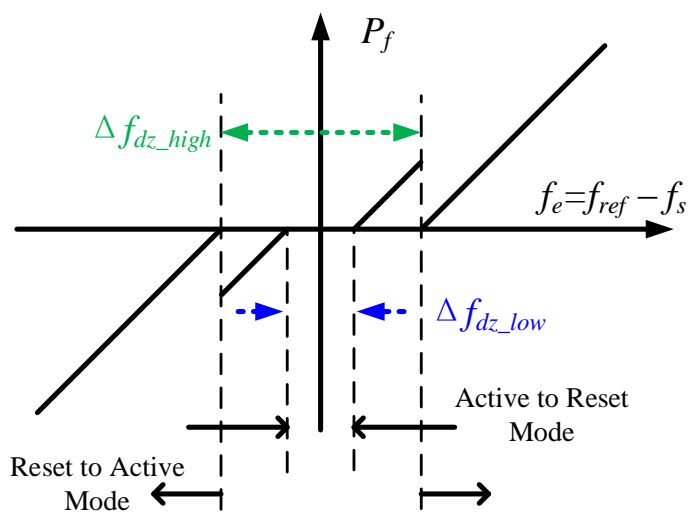

Fig. 7. Operation of the proposed dead zone control with a hysteresis band.
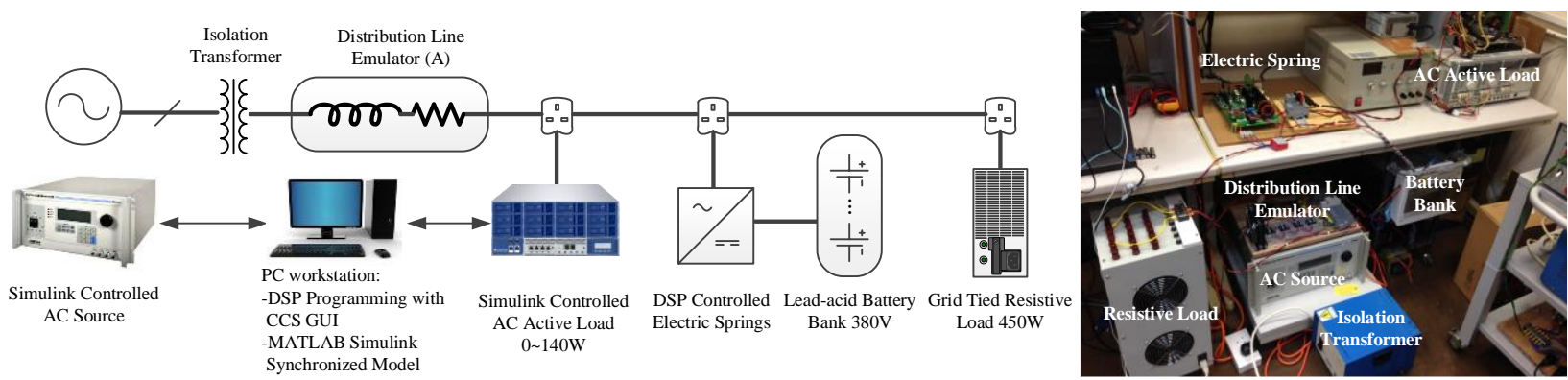

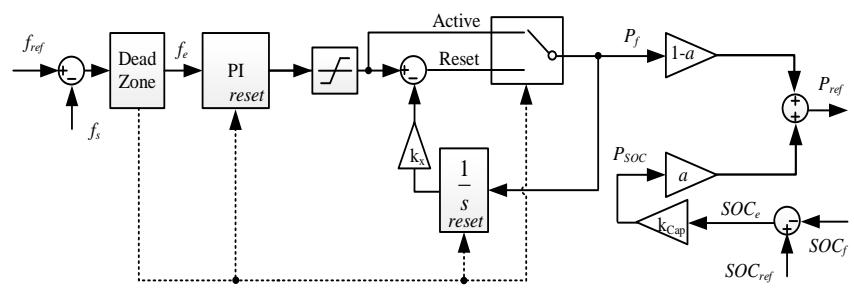

Fig. 8. Modified control scheme of frequency control loop.

\section{EXPERIMENT \& SIMULATION RESULTS}

\section{A. Experiment system setup}

A single-phase micro power system with an installed ES as shown in Fig. 9 is set up to validate the proposed coordinated battery management control scheme. A programmable AC power supply controlled by a synchronized Simulink model via IEEE 488 bus is used to emulate a generator. The frequency characteristic of this generator is based on the swing equation (8),

$$
P_{m}-P_{s}=J \omega_{r} \frac{d \omega_{r}}{d t}
$$

where $P_{m}, P_{g}, J$, and $\omega_{r}$ are the mechanical power, electrical power, rotational inertia, and the angular velocity of the rotor, respectively. Any imbalance between $P_{m}$ and $P_{g}$ could cause the generator frequency to vary. The control scheme of the generator emulator is shown in Fig. 10. Due to the hardware limitation in the communication link between Simulink and the instrument, the sample time of this model is $0.4 \mathrm{~s}$. The simplified generator emulator based on the swing equation (8) will be used for transient tests.

In the setup, the distribution line is emulated by an inductor connected in series with a power resistor, with an inductance/resistance ratio of $2 \mathrm{mH} / \Omega$. A constant resistive load and a variable AC active load controlled by Simulink are incorporated (Fig.9). A bi-directional full-bridge converter is used as the ES and is connected to a lead acid battery bank based on the control scheme shown in Fig. 5. For safety reasons, the capacity of the battery bank is sufficiently large and only a small part (namely $0.025 \mathrm{Ah}$ ) is involved in the experiment. This amount of storage capacity is set as the full SOC for calculation in this study. Fig. 11 shows the flow chart of the proposed control scheme in DSP.

\section{B. Experiment Results}

The objectives of the experiment are to analyze both the grid voltage and utility frequency regulation performance with the ES adopting the proposed control method and to validate the proposed battery management control scheme. Before the ES is tested for grid voltage and frequency regulation, the SOC

Fig. 9. Experiment system setup.

Fig. 
estimation of the battery model is first verified in order to confirm its accuracy. Then the hardware setup is tested for practical evaluation.

\section{1) Performance of the battery model}

A $12 \mathrm{~V}, 7.2 \mathrm{Ah} / 20 \mathrm{hr}$, lead acid battery cell with Model No. LC-R127R2NA is used in this part. The parameter extraction begins with the ratio of the available capacity $c$. According to the official datasheet, the rating capacity is $7.2 \mathrm{Ah}$, namely $25920 \mathrm{~A} \cdot \mathrm{s}$ when discharging with $360 \mathrm{~mA}$. Therefore, the total available capacity $y_{0}$ is confirmed. To determine the available capacity, the battery should be discharged to the cutoff voltage at a large current. According to the datasheet, a new battery could discharge 7.2A (1C) for 32 minutes. So the available capacity $y_{1,0}$ is $13824 \mathrm{~A} \cdot \mathrm{s}$. Accordingly, unavailable capacity $y_{2,0}$ is $12096 \mathrm{~A} \cdot \mathrm{s}$, and $c$ is 0.533 $(=13824 / 25920)$. In the test, the battery cell (which is fully charged and then left 24 hours for rest) discharges with $7.2 \mathrm{~A}$ in $1483 \mathrm{~s}$ (or 25 minutes) as shown in Fig. 12. Such result indicates some reduction of the capacity, which reflects the aging of the battery cell. Hence, dividing the actual availablecapacity by the typical available-capacity, the $\mathrm{SOH}$ is 0.78 . All the typical capacity values, namely $y_{0}, y_{1,0}$ and $y_{2,0}$, are multiplied by the SOH for correction. The corrected capacity values could be found in Table $\mathrm{I}$ in the appendix.

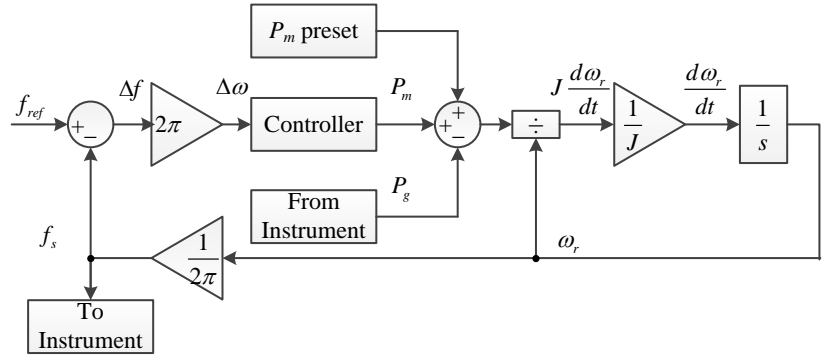

Fig.10. Control scheme of the generator emulator.

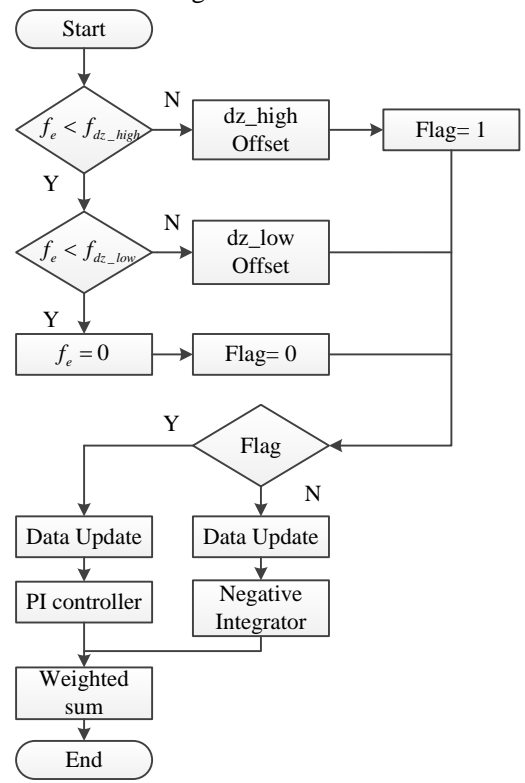

Fig.11. Flow chart of the modified frequency control scheme.

Secondly, the parameter $k$ ' in (2) could be extracted with existing measurements. It is obvious that $C_{u n}$ obtained from (1) agrees with experimental results by discharging the battery cell with constant currents from full SOC until the cutoff voltage is reached. Since $C_{u n}$ is zero at $t_{0}=0$ with sufficient rest of battery cell, $t_{d}$ is known according to measurements and the value of $c$ has been derived. Only $k^{\prime}$ remains unknown in (1). Therefore, $k^{\prime}$ can be extracted easily by a numerical method from the measurements. Although $k^{\prime}$ may vary slightly when discharging with different current, it still could be regarded as a constant for the same type of batteries [19].

To extract the parameters of the RC networks, least-square curve fitting of the experiment data is the key method used. Fig. 13 shows a typical curve of terminal voltage response used for extraction. Equation (9) provides the mathematical expression for estimating the parameters from the measured curve at the selected SOC.

$$
\left\{\begin{array}{l}
R_{\text {series }}=\frac{V_{1}-V_{0}}{I} \\
V_{\text {cell }}(t)=a\left(1-e^{-b \cdot t}\right)+c\left(1-e^{-d \cdot t}\right)+V_{1}, t_{d}<t<\infty \\
R_{\text {trans_s }}=a / I, C_{\text {trans_s }}=I / a b \\
R_{\text {trans_L }}=c / I, C_{\text {trans_L }}=I / c d
\end{array}\right.
$$

where $V_{\text {cell }}$ is the output voltage of the battery cell. The value of $V_{\text {cell }}(\infty)$ is regarded as the static output voltage of the battery $\left(V_{O C}\right)$ with the SOC at the beginning of the interval point $\left(t_{r}\right)$.

In the full discharging process, experimental data could be obtained by discharging the battery for a certain period and then letting the battery cell rest for enough time, e.g., $15 \mathrm{~min}$, to allow the voltage and part of the available capacity to recover. Hence, the parameters at the selected points against different SOC can be determined, which could be used for the final round of curve fitting to extract $a_{n}, b_{n}, c_{n}, d_{n}$ and $e_{n}$. All of these extracted parameters are tabulated in Table I in the appendix.

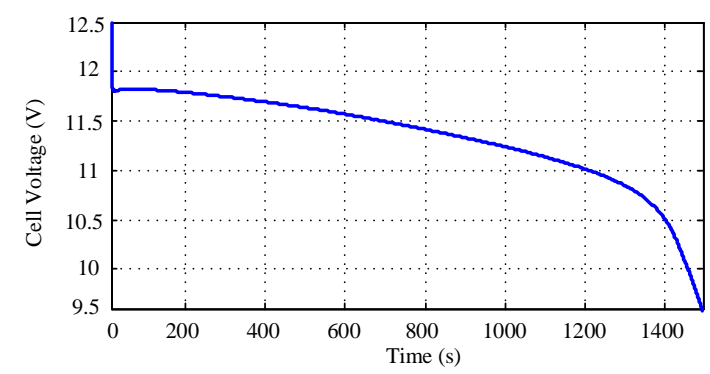

Fig. 12. Cell voltage curve when discharged with constant current of $7.2 \mathrm{~A}$.

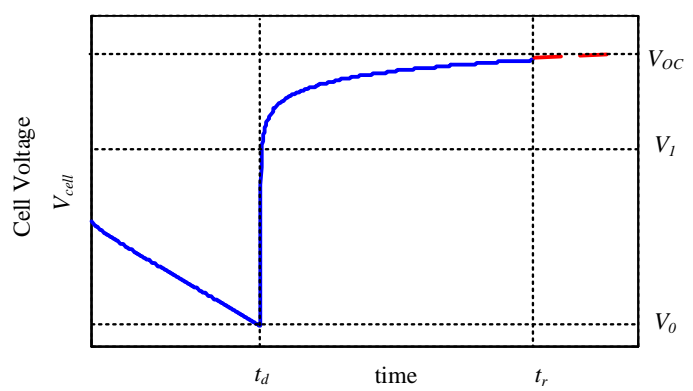

Fig. 13. A typical curve of battery output voltage with interval discharge.

Based on the hybrid model, the simulated cell voltage profile in the discharge process is plotted with the measured one in Fig. 14. The battery is discharged with $2.88 \mathrm{~A}$ for 10 minutes and left for rest for 15 minutes repeatedly until the cutoff voltage $(9.8 \mathrm{~V})$ is reached. The good agreement 
between the measured and simulated results in Fig.14 confirms the accuracy of the hybrid model. Fig. 15 shows the simulation results of the dynamic of $C_{u n}$ and SOC. These results show that the hybrid battery model is valid for full discharging process and over a wide range of SOC. The accuracy of this hybrid model gives us the confidence of using the estimated SOC as a variable in the battery management control of the ES in mitigating fluctuations in the mains voltage and frequency.

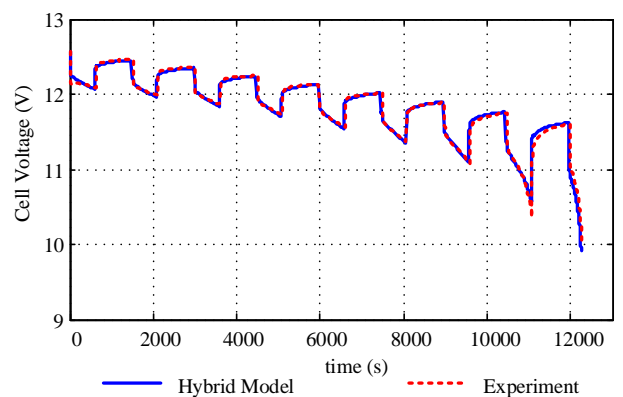

Fig. 14. Comparison of simulation and experimental curves of terminal voltage response with interval discharging.

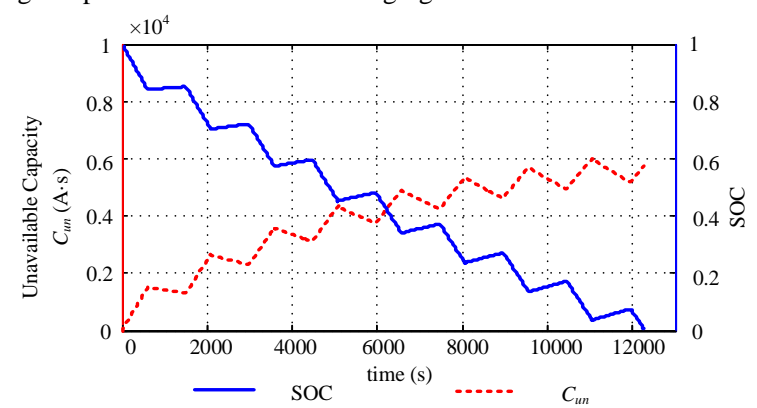

Fig. 15. Simulation results of the dynamic SOC and $C_{u n}$ with interval discharging.

\section{2) Performance on grid voltage and frequency regulation}

In the first practical test, the micro power system with a capacity of $650 \mathrm{~W}$, is initially operated at $450 \mathrm{~W}$. Then a sudden load increase of $140 \mathrm{~W}$ occurs at $t=10 \mathrm{~s}$. This test is first conducted without the ES, and is repeated with the ES activated. The practical mains voltage and frequency are captured and shown in Fig.16. The load change causes a corresponding drop in mains voltage and frequency. Such fluctuations are much larger when the ES is not activated. With the use of the ES, the mains voltage and frequency can be restored much faster and the magnitudes of the fluctuations are significantly reduced.

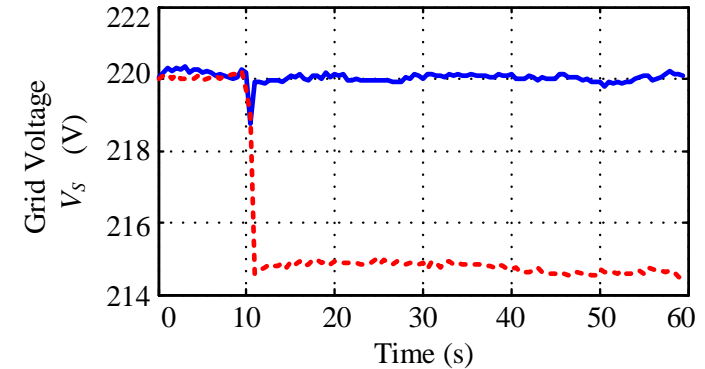

(a) Grid voltage.

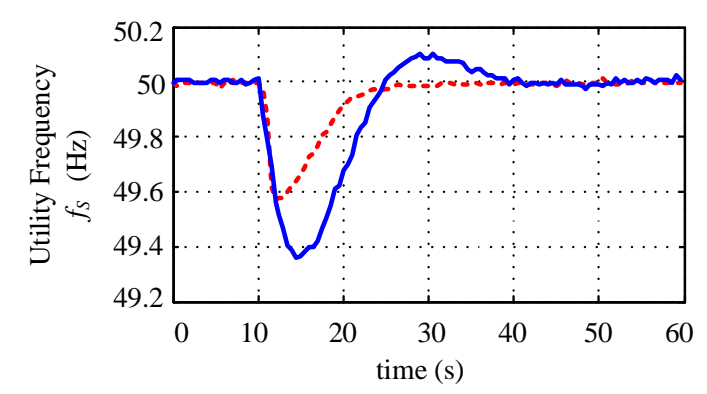

.... Without ES

(b) Utility frequency.

Fig. 16. Experimental results from $\mathrm{t}=0$ to $60 \mathrm{~s}$.

\section{3) Proposed battery management control scheme}

After the transient response by the ES to restore the mains voltage and frequency after the sudden load change, the battery management control takes actions to gradually regulate the SOC to 0.5 . Fig. 17 shows a full test period from $t=0$ to $1400 \mathrm{~s}$. The ES active power is expressed in percentage based on the active power limit $( \pm 180 \mathrm{~W}$, or \pm 0.277 p.u.). At $t=10 \mathrm{~s}$, the ES starts to deliver active power of around $68 \%$ ( 0.188 p.u.) to cope with the sudden change of load power at the demand side. The grid frequency is corrected into the lower deviation range, namely $50 \pm 0.02 \mathrm{~Hz}$ at $t=25 \mathrm{~s}$ (Fig. 16(b)) and the active power control of the ES is switched to reset mode. During $t=25 \mathrm{~s}$ to $t \approx 300 \mathrm{~s}$, the reset integrator (Fig. 8) is in action, causing $P_{e s}$ to approach zero. The rate of reduction of $P_{e s}$ during this period is controlled by $k_{x}$. The tuning of the positive parameter $k_{x}$ is related to the original frequency response of the grid. For example, if a grid (without ES installed) takes a long time to recover from a certain frequency drop, the $k_{x}$ should be decreased so that $P_{e s}$ approaches zero smoothly in order to avoid potential chattering. As the $S O C_{f}$ keeps deviating from $S O C_{r e f}=0.5$, the weighted factor $a$ increases, which forces the system to charge the battery more aggressively. Hence, after $t \approx 300 \mathrm{~s}, P_{e s}$ becomes negative and $S O C_{f}$ keeps increasing. As $S O C_{f}$ approaches 0.5 , the charging power is significantly reduced due to the reduction of the weighted factor $a$, which results in a much longer period to reach 0.5. Compared with the original

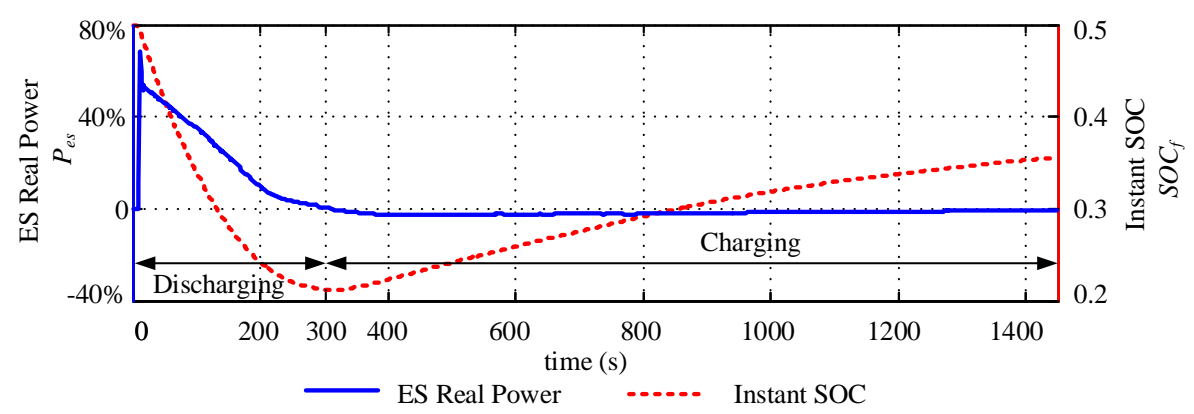

Fig.17. Experimental results from $\mathrm{t}=0$ to $1400 \mathrm{~s}$. 
active power flow of ES as shown in Fig. 4(b), the active power flow of ES with proposed control scheme (i) is needed for stabilizing the grid voltage and frequency during the transient period and (ii) is gradually reduced to zero in the reset mode while restoring the SOC to 0.5 . The battery management is targeted for this purpose and the battery is not used for providing active power continuously for long period of time. This feature differentiates its application from the traditional use of battery in a grid-connected PV system. Consequently, batteries with small capacity can be used for this ES application under the proposed control scheme.

\section{Microgrid Simulation Study}

To further validate the proposed control scheme at a higher power level, a microgrid model is developed in Simulink as shown in Fig. 18. In this model, a synchronous generator (5 MVA) with excitation control is driven by a steam turbine with a governor. The full specifications of the generator model are provided in the appendix. The demand side is connected to the generation side via a $5 \mathrm{~km}$ distribution line and a stepdown transformer. The battery bank, which has an initial SOC of 0.5 with a full capacity of $80 \mathrm{kWh}$, is constructed with 28 parallel battery packs. Each battery pack consists of 30 seriesconnected battery cells. The limits of the active and reactive power outputs are $250 \mathrm{~kW}$ and $500 \mathrm{kVar}$ respectively. In the simulation study, the switch $\mathrm{S} 1$ is closed at $t=4 \mathrm{~s}$ to cause a sudden change of the load power $(0.12$ p.u. $)$ on the demand side.

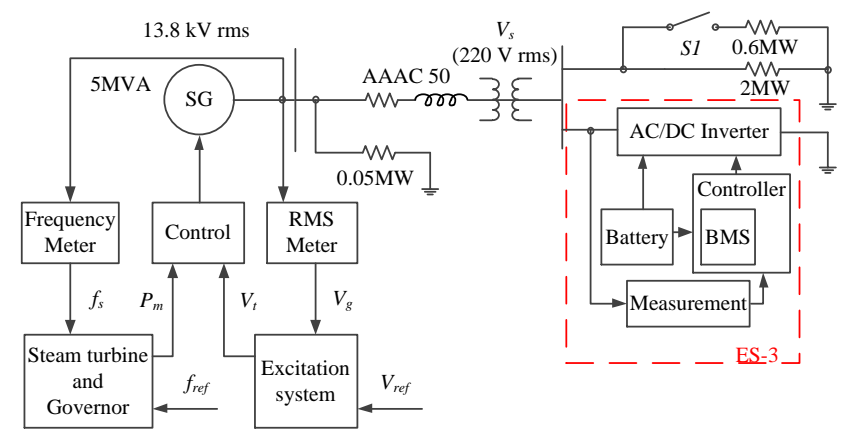

Fig.18. Simulation diagram of microgrid system with ES-3 installed.

Fig. 19 shows the simulation results of the grid voltage and frequency from $t=0$ to $60 \mathrm{~s}$. The results indicate that the control scheme is also effective in high power level. The simulation results with a duration of $1500 \mathrm{~s}$ for the proposed battery management control scheme are shown in Fig. 20. As the active power of the ES is limited to $250 \mathrm{~kW}$, the peak value of the active power delivered from the ES at $t=4 \mathrm{~s}$ is around $75 \%$ ( 0.0375 p.u.) for compensating the frequency drop. The
ES enters into the reset mode as the frequency error reaches the preset small deviation range at $t=20 \mathrm{~s}$. Similar to the experimental results, after $t \approx 220 \mathrm{~s}, P_{e s}$ becomes negative and the battery is being charged. With the similar performance observed from the experimental result, the simulation results indicate that the proposed control scheme is applicable at a high power level for providing grid stability enhancement.

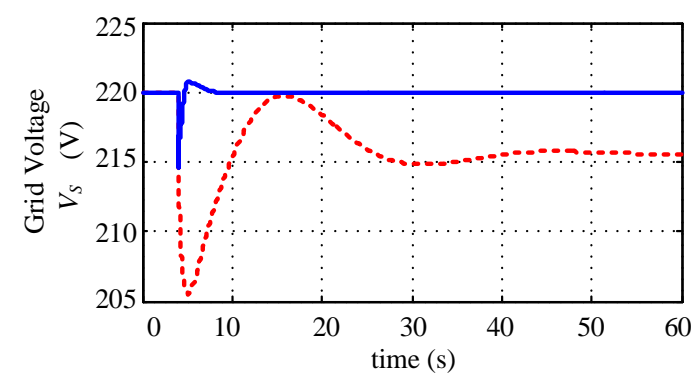

(a) Grid voltage.

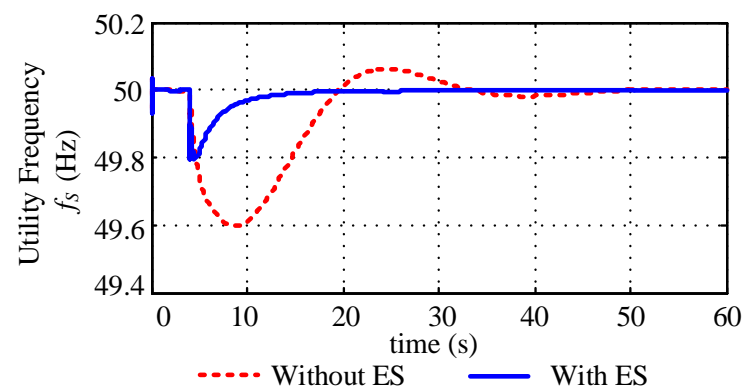

(b) Utility frequency.

Fig.19. Simulation results from $t=0$ to $60 \mathrm{~s}$.

\section{CONCLUSIONS}

In this project, the third version of the electric spring (based on the use of a gird-connected bidirectional power converter and battery) is operated with a coordinated battery management system. A new control scheme with a new stateof-charge control algorithm is proposed to operate the battery within a SOC range that will not degrade the lifetime of the battery. The proposed scheme has been practically verified with a realistic hybrid battery model with accurate SOC estimation. A modified dead zone control circuit with a hysteresis band embedded on the existing active-reactive power decoupling control scheme for ES-3 has been successfully demonstrated. Experimental and simulation results have indicated that the proposed control method preserves the original functions of ES-3 on both grid voltage and frequency regulation while providing an effective way for managing a healthy state-of-charge of the battery system. With the automatic charging and discharging strategy integrated in the active power control loop, many bidirectional

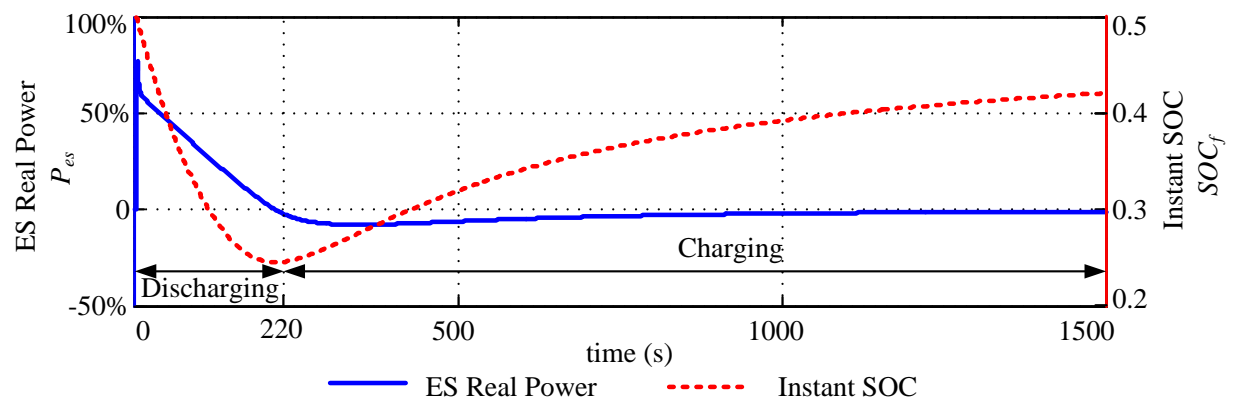

Fig. 20. Simulation results from $\mathrm{t}=0$ to $1500 \mathrm{~s}$. 
ES with limited energy storage capacities can in principle be equipped with the electric spring functions. They can play an important role collectively as distributed devices to provide active and reactive power compensation for improving the stability of future power grid.

\section{APPENDIX}

TABLE I.

Extracted Battery Model Parameters

\begin{tabular}{|c|c|c|c|c|c|}
\hline $\boldsymbol{y}_{\boldsymbol{\theta}}$ & $\boldsymbol{y}_{\boldsymbol{1 , \boldsymbol { \theta }}}$ & $\boldsymbol{y}_{\boldsymbol{2}, \boldsymbol{\theta}}$ & $\boldsymbol{c}$ & $\boldsymbol{k}^{\prime}$ & $\boldsymbol{S O H}$ \\
\hline $\begin{array}{c}20476 . \\
8\end{array}$ & $\begin{array}{c}10920.9 \\
6\end{array}$ & $\begin{array}{c}9555.8 \\
4\end{array}$ & 0.533 & 0.0001 & 0.78 \\
\hline $\boldsymbol{a}_{\boldsymbol{0}}$ & $\boldsymbol{a}_{\boldsymbol{1}}$ & $\boldsymbol{a}_{\boldsymbol{2}}$ & $\boldsymbol{a}_{\boldsymbol{3}}$ & $\boldsymbol{a}_{\boldsymbol{4}}$ & $\boldsymbol{a}_{5}$ \\
\hline-1.0262 & $\begin{array}{c}249.937 \\
0\end{array}$ & $\begin{array}{c}11.493 \\
7\end{array}$ & 1.7477 & 1.0154 & 0.3423 \\
\hline $\boldsymbol{b}_{\boldsymbol{0}}$ & $\boldsymbol{b}_{\boldsymbol{1}}$ & $\boldsymbol{b}_{\boldsymbol{2}}$ & $\boldsymbol{b}_{\boldsymbol{3}}$ & $\boldsymbol{b}_{\boldsymbol{4}}$ & $\boldsymbol{b}_{\boldsymbol{5}}$ \\
\hline 0.2202 & 58.3115 & 0.1614 & -0.3474 & -0.3904 & -0.1383 \\
\hline $\boldsymbol{c}_{\boldsymbol{\theta}}$ & $\boldsymbol{c}_{\boldsymbol{1}}$ & $\boldsymbol{c}_{\boldsymbol{2}}$ & $\boldsymbol{d}_{\boldsymbol{0}}$ & $\boldsymbol{d}_{\boldsymbol{1}}$ & $\boldsymbol{d}_{\boldsymbol{2}}$ \\
\hline 0.1137 & 11.6634 & 0.0372 & - & 7.8288 & 260.4930 \\
\hline $\boldsymbol{e}_{\boldsymbol{0}}$ & $\boldsymbol{e}_{\boldsymbol{1}}$ & $\boldsymbol{e}_{\boldsymbol{2}}$ & $\boldsymbol{f}_{\boldsymbol{0}}$ & $\boldsymbol{f}_{\boldsymbol{1}}$ & $\boldsymbol{f}_{\boldsymbol{2}}$ \\
\hline 0.4275 & 55.2052 & 0.0360 & -0.0086 & 6.1542 & 0.0094 \\
\hline
\end{tabular}

TABLE II.

Simulink Model Synchronous Machine Specifications

\begin{tabular}{|c|c|}
\hline \multicolumn{2}{|c|}{ Synchronous Machine } \\
\hline Rotor Type & Round \\
\hline $\begin{array}{l}\text { Nominal power, line to line voltage and } \\
\text { frequency }[\operatorname{Pn}(\mathrm{VA}), \mathrm{Vn}(\mathrm{Vrms}), \mathrm{fn}(\mathrm{Hz})]\end{array}$ & {$\left[5 \times 10^{6}, 13800,50\right]$} \\
\hline $\begin{array}{c}\text { Reactaces } \\
{[\mathrm{Xd}, \mathrm{Xd} \text { ',Xd",,Xq,Xq',Xq", Xl](p.u.) }}\end{array}$ & $\begin{array}{c}{[1.650 .25,0.2,1.59,0.46,} \\
0.2,0.14]\end{array}$ \\
\hline $\begin{array}{c}\text { Time constants } \\
\text { [Tdo', Tdo", Tqo', Tqo"] }\end{array}$ & {$[4.5,0.04,0.67,0.09]$} \\
\hline Stator resistance $\operatorname{Rs}()$ & 0.0045 \\
\hline $\begin{array}{l}\text { Inertia coefficient, friction factor, pole } \\
\text { pairs }[\mathrm{H}(\mathrm{s}), \mathrm{F}(\text { p.u. }), \mathrm{p}()]\end{array}$ & {$[3.2,0,1]$} \\
\hline
\end{tabular}

TABLE III.

Simulink Model Excitation System Specifications Excitation System

\begin{tabular}{|c|c|}
\hline \multicolumn{2}{|c|}{ Excitation System } \\
\hline \multicolumn{2}{|c|}{ Low-pass filter time constant $\mathrm{Tr}(\mathrm{s})$} \\
\hline $\begin{array}{c}\text { Regulator gain and time constant }[\mathrm{Ka}(), \\
\mathrm{Ta}(\mathrm{s})]\end{array}$ & 0.020 \\
\hline Exciter $[\mathrm{Ke}(), \mathrm{Te}(\mathrm{s})]$ & {$[1000,0.01]$} \\
\hline $\begin{array}{c}\text { Transient gain reduction }[\mathrm{Tb}(\mathrm{s}), \mathrm{Tc}(\mathrm{s})] \\
\text { Damping filter gain and time constant } \\
{[\mathrm{Kf}(), \mathrm{Tf}(\mathrm{s})]}\end{array}$ & {$[0,0]$} \\
\hline $\begin{array}{c}\text { Regulator output limits and gain }[\mathrm{Efmin}, \\
\text { Efmax(p.u.), Kp()] }\end{array}$ & {$[-11.5,11.50]$} \\
\hline
\end{tabular}

TABLE IV

Simulink Model Steam Turbine \& Governor Specifications

\begin{tabular}{|c|c|}
\hline \multicolumn{2}{|c|}{ Steam Turbine and Governor } \\
\hline Generator type & $\begin{array}{c}\text { Tandem-compound (single } \\
\text { mass) }\end{array}$ \\
\hline $\begin{array}{c}\text { Regulator gain, perm. Droop, dead zone } \\
\text { [Kp, Rp(p.u.), Dz(p.u.)] }\end{array}$ & {$[1,0.08,0]$} \\
\hline $\begin{array}{c}\text { Gate opening limites [vgmin, } \\
\text { vgmax(p.u./s), gmin, gmax(p.u.)] }\end{array}$ & {$[-0.2,0.2,0,1]$} \\
\hline $\begin{array}{c}\text { Nominal speed of synchronous } \\
\text { machine(rpm) }\end{array}$ & 3000 \\
\hline $\begin{array}{c}\text { Steam turbine time constant [T2, T3, T4, } \\
\text { T5](s) }\end{array}$ & {$[0,10,3.3,0.5]$} \\
\hline Turbine torque fractions [F2, F3, F4, F5] & {$[0,0.36,0.36,0.28]$} \\
\hline
\end{tabular}

TABLE V.

Simulink Model Transformer Specifications Transformer

\begin{tabular}{|c|c|}
\hline \multicolumn{2}{|c|}{ Transformer } \\
\hline $\begin{array}{c}\text { Windings connection } \\
\text { Nominal power and frequency [Pn(VA), } \\
\text { fn(Hz)] }\end{array}$ & Delta to Star ground \\
\hline $\begin{array}{c}\text { Winding 1 parameters [V1 Ph-Ph(Vrms), } \\
\text { R1(p.u.), L1(p.u.)] }\end{array}$ & {$\left[132610^{6}, 50\right]$} \\
\hline $\begin{array}{c}\text { Winding 2 parameters [V2 Ph-Ph(Vrms), } \\
\text { R2(p.u.), L2(p.u.)] }\end{array}$ & {$[381,0.00270 .08]$} \\
\hline $\begin{array}{c}\text { Magnetization resistance Rm(p.u.) } \\
\text { Magnetization resistance Lm(p.u.) }\end{array}$ & 500 \\
\hline
\end{tabular}

\section{ACKNOWLEDGMENT}

This project is supported by the Hong Kong Research Grant Council under a Theme-based Project: T23-701/14-N.

\section{VIII.REFERENCES}

[1] A. H. Mohsenian-Rad, V. W. S. Wong, J. Jatskevich, R. Schober, and A. Leon-Garcia, "Autonomous demand-side management based on game-theoretic energy consumption scheduling for the future smart grid," IEEE Trans. Smart Grid, vol. 1, no. 3, pp. 320-331, Dec. 2010.

[2] F. Kienzle, P. Ahcin, and G. Andersson, "Valuing investments in multienergy conversion, storage, and demand-side management systems under uncertainty," IEEE Trans. Sustain. Energy, vol. 2, no. 2, pp. 194 202, Apr. 2011.

[3] A. J. Conejo, J. M. Morales and L. Baringo, "Real-time demand response model," IEEE Trans. Smart Grid, vol. 1, no. 3, pp. 236-242, Dec. 2010 .

[4] S. C. Lee, S. J. Kim, and S. H. Kim, "Demand side management with air conditioner loads based on the queuing system model," IEEE Trans. Power Syst., vol. 26 , no. 2, pp. 661-668, May 2011.

[5] J.M. Guerrero, J.C. Vasquez, J. Matas, M. Castilla and L. Garcia de Vicuna, "Control strategy for flexible microgrid based on parallel lineinteractive UPS systems", IEEE Transactions on Industrial Electronics, Vol. 56, No.3, March 2009, pp: 726-735

[6] P. Khayyer and U. Ozguner, "Decentralized control of large-scale storage-based renewable energy systems", IEEE Transactions on Smart Grid, Vol. 5, No.3, May 2014, pp: 1300-1307.

[7] A. Mohamed, V. Salehi and O. Mohammed, "Real-time energy management algorithm for mitigation of pulse loads in hybrid microgrids", IEEE Transactions on Smart Grid, Vol. 3, No.4, December 2012, pp: 1911-1922.

[8] N.Tummuru, M. Mishra and S. Srinivas, "Integration of PV/battery hybrid energy conversion system to the grid with power quality improvement features", IEEE International Conference on Industrial Technology (ICIT) 2013, Cape Town, 25-28 Feb., 2013, pp: 1751-1756

[9] A. Esmaili, B. Novakovic, A. Nasiri and O. Abdel-Baqi, "A hybrid system of Li-ion capacitors and flow battery for dynamic wind energy support", IEEE Transactions on Industry Applications, Vol. 49, No. 4, July/August 2013, pp: 1649-1657

[10] S. Y. R. Hui, C. K. Lee, and F. F. Wu, "Electric springs - a new smart grid technology," IEEE Trans. Smart Grid, vol. 3, no. 3, pp. 1552 1561, Sep. 2012.

[11] S. C. Tan, C. K. Lee, and S. Y. R. Hui, "General steady-state analysis and control principle of electric springs with active and reactive power compensations," IEEE Trans. Power Electron., vol. 28, no. 8, pp. 39583969, Aug. 2013.

[12] S. Yan, S. C. Tan, C. K. Lee, and S. Y. R. Hui, "Electric spring for power quality improvement," IEEE Applied Power Electronics Conference and Exposition (APEC), pp. 2140-47, Mar. 2014.

[13] C. K. Lee and S. Y. R. Hui, "Input voltage control bidirectional power converters," US patent application, US2013/0322139, filed 31 May 2013.

[14] Tianbo Yang, Kwan-Tat Mok, Siew-Chong Tan and S.Y.Ron Hui, "Control of electric springs with coordinated battery management", IEEE Energy Conversion Congress and Exposition (ECCE), 2015, pp: $6740-6746$

[15] M. Doyle, T. Fuller and J. Newman, "Modeling of galvanostatic charge and discharge of the Lithium/Polymer/Insertion cell", Journal of the Electrochemical Society, Vol. 140, No.6, June 1993, pp: 1526-1533

[16] V. Pop, et la, "Modeling battery behavior for accurate state-of-charge indication", Journal of the Electrochemical Society, Vol. 153, No. 11, 2006, pp: A2013-A2022 
[17] H. Fang, Y. Wang, Z. Sahinoglu, T. Wada and S. Hara, "Adaptive estimation of state of charge for lithium-ion batteries", 2013 American Control Conference, Washington, DC, USA, June 17-19, 2013, pp: 3485-3491

[18] M. Chen and G. Rincon-Mora, "Accurate electrical battery model capable of predicting runtime and I_V performance", IEEE Transactions on Energy Conversion, Vol. 21, No.2, June 2006, pp: $504-$ 511

[19] J. Zhang, S. Ci, H. Sharif and M. Alahmad, "An enchanced circuitbased model for single-cell battery", 25th Annual IEEE Applied Power Electronics Conference (IEEE APEC), 21-25 Feb. 2010, Palm Springs, USA, 2010, pp: 672-675.

[20] T. Kim and W. Qiao, "A hybrid battery model capable of capturing dynamic circuit characteristics and nonlinear capacity effects," IEEE Transactions on Energy Conversion, vol. 26, no. 4, pp. 1172-1180, Dec. 2011.

[21] Tummuru, N.R., M.K. Mishra, and S. Srinivas. "Dynamic Energy Management of Renewable Grid Integrated Hybrid Energy Storage System." IEEE Transactions on Industrial Electronics, vol. 62, no. 12 Dec 2015, pp: 7728-7737.

[22] Eremia, Mircea, and Mohammad Shahidehpour, "Handbook of Electrical Power System Dynamics: Modeling, Stability, and Control," John Wiley \& Sons, 2013.

\section{BIOGRAPHIES}

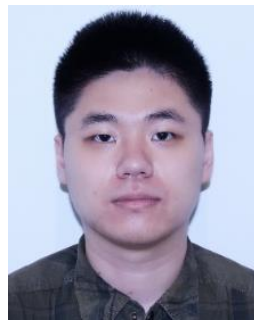

Tianbo Yang (S'15) received the B.Eng. and M.Phil. degrees in automation and control engineering from the Harbin Institute of Technology, China, in 2012 and 2014, respectively. He is currently pursuing the $\mathrm{Ph} . \mathrm{D}$. degree at the Department of Electrical and Electronic Engineering, the University of Hong Kong. His research interest includes power electronic technologies in smart grid and energy storage system.

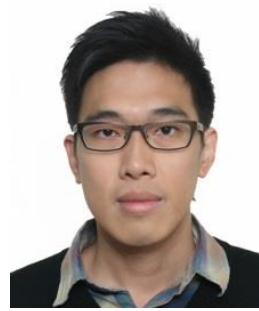

Kwan-Tat Mok (S'14) received the B.Eng. (Hons.) and M.Phil degrees in electronic and information engineering from the Hong Kong Polytechnic University, in 2009 and 2012, respectively. In 2007, he worked as an Engineering Trainee in Solomon Systech Limited, Hong Kong, for a one-year internship program. From Septembe 2011 to February 2013, he was an Embedded System Engineer in Cwlinux Limited, Hong Kong. From July to August 2015, he was a visiting postgraduate student in Department of Electrical and Electronic Engineering, Imperial College London. He is currently a Ph.D. candidate in Power Electronics Research Group, Department of Electrical and Electronic Engineering, The University of Hong Kong. His research interests include smart grid technologies, electric springs and power converters for lightemitting diodes.

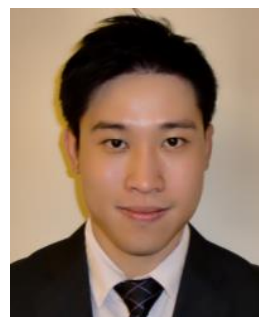

Siew-Chong Tan (M'06-SM'11) received the B.Eng. (Hons.) and M.Eng. degrees in electrical and computer engineering from the National University of Singapore, Singapore, in 2000 and 2002, respectively, and the Ph.D. degree in electronic and information engineering from the Hong Kong Polytechnic University, Hong Kong, in 2005.

From October 2005 to May 2012, he worked as Research Associate, Postdoctoral Fellow, Lecturer, and Assistant Professor in Department of Electronic and Information Engineering, Hong Kong Polytechnic University, Hong Kong. From January to October 2011, he was Senior Scientist in Agency for Science, Technology and Research (A*Star), Singapore. He is currently an Associate Professor in Department of Electrical and Electronic Engineering, The University of Hong Kong, Hong Kong. Dr. Tan was a Visiting Scholar at Grainger Center for Electric Machinery and Electromechanics, University of Illinois at Urbana-Champaign, Champaign, from September to October 2009, and an Invited Academic Visitor of Huazhong University of Science and Technology, Wuhan, China, in December 2011. His research interests are focused in the areas of power electronics and control, LED lightings, smart grids, and clean energy technologies.

Dr. Tan serves extensively as a reviewer for various IEEE/IET transactions and journals on power, electronics, circuits, and control engineering. He is an Associate Editor of the IEEE Transactions on Power Electronics. He is a coauthor of the book Sliding Mode Control of Switching
Power Converters: Techniques and Implementation (Boca Raton: CRC 2011)

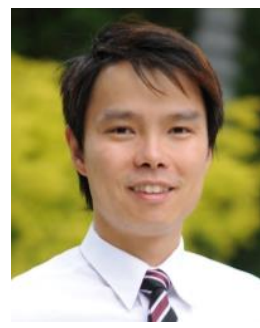

Chi Kwan Lee (M'08-SM'14) received the B.Eng. and $\mathrm{Ph} . \mathrm{D}$. degrees in electronic engineering from the City University of Hong Kong, Kowloon, Hong Kong, in 1999 and 2004, respectively.

$\mathrm{He}$ is currently an Assistant Professor at the Department of Electrical \& Electronic Engineering, The University of Hong Kong. Since 2010, He has been a Visiting Researcher with Imperial College London.

Dr. Lee was a Postdoctoral Research Fellow in the Power and Energy Research Centre at the National University of Ireland, Galway, from 2004 to 2005. In 2006, he joined the Centre of Power Electronics in City University of Hong Kong as a Research Fellow. In 20082011 he was a Lecturer of Electrical Engineering at the Hong Kong Polytechnic University. He won an IEEE Power Electronics Transactions First Prize Paper Award for his publications on Mid-Range Wireless Power Transfer in 2015. He is a co-inventor of the Electric Springs and planar EMI filter. His current research interests include wireless power transfer, clean energy technologies and smart grids.

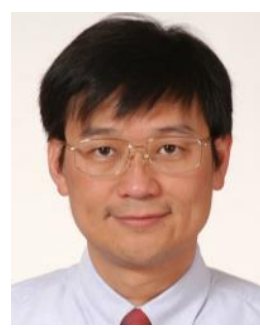

S. Y. (Ron) Hui (M'87-SM'94-F'03) received his B.Sc. (Eng. Hons.) from the University of Birmingham in 1984 and the D.I.C. and Ph.D. degrees from Imperial College London, London, U.K., in 1987.

He has previously held academic positions at the University of Nottingham (1987-1990), University of Technology Sydney (1991-1992), University of Sydney (1992-1996), City University of Hong Kong (1996-2011). Since July 2011, he has been a Chair Professor at the University of Hong Kong. He has been a part-time Chair Professor at Imperial College London since 2010. He has published more than 300 technical papers, including more than 220 refereed journal publications and book chapters. Over 60 of his patents have been adopted by industry.

Dr. Hui has been an Associate Editor of the IEEE TRANSACTIONS ON POWER ELECTRONICS since 1997 and an Associate Editor of the IEEE TRANSACTIONS ON INDUSTRIAL ELECTRONICS since 2007. He is an Editor of the IEEE JOURNAL OF EMERGING AND SELECTED TOPICS OF POWER ELECTRONICS. He was appointed twice as an IEEE Distinguished Lecturer by the IEEE Power Electronics Society in 2004 and 2006. In 2010, he received the IEEE Rudolf Chope R\&D Award from the IEEE Industrial Electronics Society, and the IET Achievement Medal (The Crompton Medal) $\mathrm{He}$ is the recipient of the 2015 IEEE Technical Field Award (IEEE William E. Newell Power Electronics Award). He is a Fellow of the Australian Academy of Technological Sciences \& Engineering and also the Royal Academy of Engineering (U.K.) 